\title{
Application of the Probability Model for Starting Period to Initiate to Take Palivizumab by Prefecture Using National Official Sentinel Surveillance in Japan
}

\author{
Junko Kurita1 ${ }^{*}$, Tamie Sugawara ${ }^{2}$, Yasushi Ohkusa², Michiko Nohara3 \\ ${ }^{1}$ Prefectural University of Health Sciences, Ami, Ibaraki, Japan \\ ${ }^{2}$ National Institute of Infectious Diseases, Tokyo, Japan \\ ${ }^{3}$ Tokyo Kasei University, Tokyo, Japan \\ Email: ^kuritaj@ipu.ac.jp
}

How to cite this paper: Kurita, J., Sugawara, T., Ohkusa, Y. and Nohara, M. (2020) Application of the Probability Model for Starting Period to Initiate to Take Palivizumab by Prefecture Using National Official Sentinel Surveillance in Japan. Journal of Biosciences and Medicines, 8, 56-63.

https://doi.org/10.4236/jbm.2020.81007

Received: November 11, 2019

Accepted: January 6, 2020

Published: January 9, 2020

Copyright $\odot 2020$ by author(s) and Scientific Research Publishing Inc. This work is licensed under the Creative Commons Attribution International License (CC BY 4.0).

http://creativecommons.org/licenses/by/4.0/

\begin{abstract}
Background: Palivizumab were used for the premature infant or a high-risk infant with congenital heart disease. However, recently outbreak pattern of respiratory syncytial (RS) virus infection has been varying year by year. Moreover, it also has some regional difference. Therefore, the object of the present study was to develop early detection of the timing of that outbreak had started in each prefecture. Method: We used data in National Official Sentinel Surveillance for Infectious Diseases (NOSSID). Study period was March 16th, 2011 to December 30th, 2018. We defined stating period to initiate to take palivizumab as $8-12$ weeks before from the peak of outbreak. We estimated whether the week is included in starting period or not from April 1st to the peak of outbreak by the past number of patients of RS virus infection on week and its squared. Additionally, we have to take delay in NOSSID into consideration. Results: In nationwide, the last two seasons, the model predicted precisely the starting period. At prefectural level, the model predicted the starting period precisely in $16.6 \%$ of all year and prefectures pairs. When we consider the delay in NOSSID into consideration, the patients can start to take in $14.9 \%$ of all year and prefectures pairs. Discussion and Conclusion: The result of the probability model was not good, and thus we have to develop more sophisticated model for prediction at prefecture level.
\end{abstract}

\section{Keywords}

Respiratory Syncytial Virus Infection, National Official Sentinel Surveillance Palivizumab, Probability Model, Starting Period 


\section{Introduction}

As generally known, even though respiratory syncytial (RS) virus infection is very common infectious disease for age 0 - 1, but its vaccination for RS virus infection was not available [1] [2] [3]. Instead of that, palivizumab were used for the premature infant or a high-risk infant with congenital heart disease. The targeted population need to shot it in every month during outbreak. However, recently outbreak pattern of RS virus infection has been varying year by year. Moreover, it also has substantial regional variation [4]. Therefore, the object of the present study was to develop early detection of the timing of that outbreak had started in each prefecture.

Recently, the probability model for starting period to initiate to take palivizumab was examined [5]. It used ORCA surveillance, which is daily reporting system for influenza, hand-foot-and-mouth disease, Erythema infectiosum, Pharyngoconjunctival fever, RS virus infection, measles, rubella and insolation from ORCA [6], which is an electronic medical claim system (http://infect.orca.med.or.jp/). ORCA was abbreviation for "Online Receipt Computer Advantage" which is online medical claim service produced and provided medical facilities freely by Japan Medical Association. ORCA was utilized in 17,174 medical facilities as of the end of September in 2019.

Even though the proposed probability model works well in nationwide, ORCA surveillance for RS cannot be break down to prefecture level due to limitation of reporting medical facilities. It reminded as about 200 facilities, and thus the probability model cannot work at prefecture level [5]. In Japan, national official surveillance for influenza has been performing at about 3000 sentinels, which is almost one of tenth of pediatric medicine, based on the Law Related to the Prevention of Infectious Diseases and Medical Care for Patients of Infections (The Infectious Diseases Control Law). We call it National Official Sentinel Surveillance for Infectious Diseases (NOSSID) [7]. However, since it reported the situation in the last week on Friday noon, it delays to report in public for 13 days at maximum. For detection of outbreak of RS virus infection, this delay might cause serious problem to initiate to take palivizumab. On the other hand, it was operated base on the law and thus it works well all prefectures in Japan. Because he previous research found that regional variation in outbreaks of RS among prefectures [4], prediction model by prefecture was necessary.

Therefore, we examined some feasibility and availability of earlier prediction model for outbreak of RS virus infection by prefecture using information of NOSSID.

\section{Method}

Study period was March 16th 2011 to December 30th, 2018. We defined stating period to initiate to take palivizumab as $8-12$ weeks before from the peak of outbreak. We dropped the data from the peak to the end of following March. We estimated whether week $t$ is included starting period or not from April 1st to the 
peak of outbreak by the number of patients of RS virus infection on week $t-k$ $(k=0,1,2, \ldots, N)$, and its squared [5]. The largest lag of number of patient, $N$, was determined by that the coefficient of the number of patients on $t-N$ was significant but insignificant on $t-N$ from $N=0$ step wisely. We applied the same specification, $N$, which is selected in the nationwide data to the prefectural data. We adopted $5 \%$ as significance level.

Then we calculated probability of starting period based on the estimation results. We suppose the criterion if the estimated probability was higher than this criterion, the model predicts the starting period on the week. The criterion was defined in each prefecture as the minimum the highest probability in each season among all five seasons. If the estimated probabilities in one week were the highest, the week was identified as the model predicted starting date. If the estimated probabilities in several weeks were higher than criterion, the first date was identified as the model predicted starting date. We identified the model was precise if predicted starting date locates during $t-12$ to $t-8$. Also we identified that it was too early if predicted starting date was before $t-12$, and too delay if predicted starting date was before $t-8$.

Additionally, we have to take delay in NOSSID as described above into consideration. The information in NOSSID on week $t$ will be available on the week $t+2$. Therefore, so as to start to take the drug during $t-12$ to $t-8$ weeks before the peak, the model should announce it on $t-14$ to $t-10$ weeks before the peak.

Season for RS virus infection was defined as from 1st April to the end of March in the following year. However, the end of season in 2018/2019 season was terminated on 30th December, 2018 due to data availability.

\section{Ethical Considerations}

Data from NOSSID was aggregated and de-linked from personal information related to patients and medical facilities: these are anonymous data. Moreover, these were published in general. Therefore, no ethical issues are posed by using these data for this study.

\section{Results}

Figure 1 showed the number of patients with RS virus infection to NSSID in nationwide. We selected $N=0$ because the number of patient on $t-1$ week were not significant in nationwide data. The estimated coefficient for number of patients on week $t$ was $0.003(0.000)$, for its squared was $-6.08 * 10^{-7}(0.000)$ and constant was $-3.28(0.000)$. The number in parenthesis was $p$-value for each explanatory variable. Figure 2 showed estimated probability of the starting period in nationwide.

The identified criteria nationwide and by prefecture were summarized at the second column in Table 1. Moreover, Table 1 contains results of model prediction and actual starting period as "A", "B" and " $\mathrm{H}$ ". " $\mathrm{H}$ " indicates the probability 


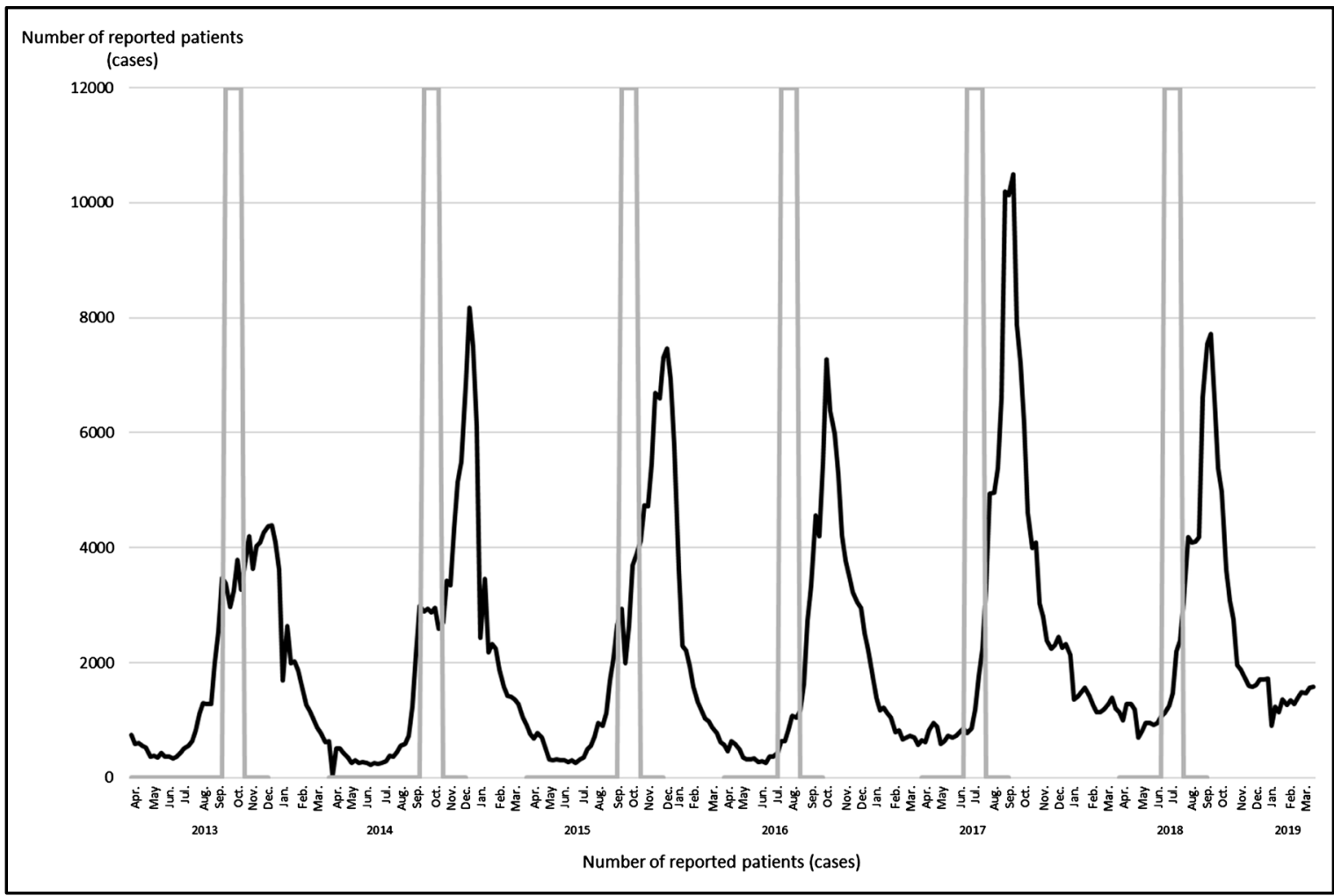

Note: The rectangular area indicate starting period in each season.

Figure 1. The number of patients with RS virus infection to NSSID in nationwide.

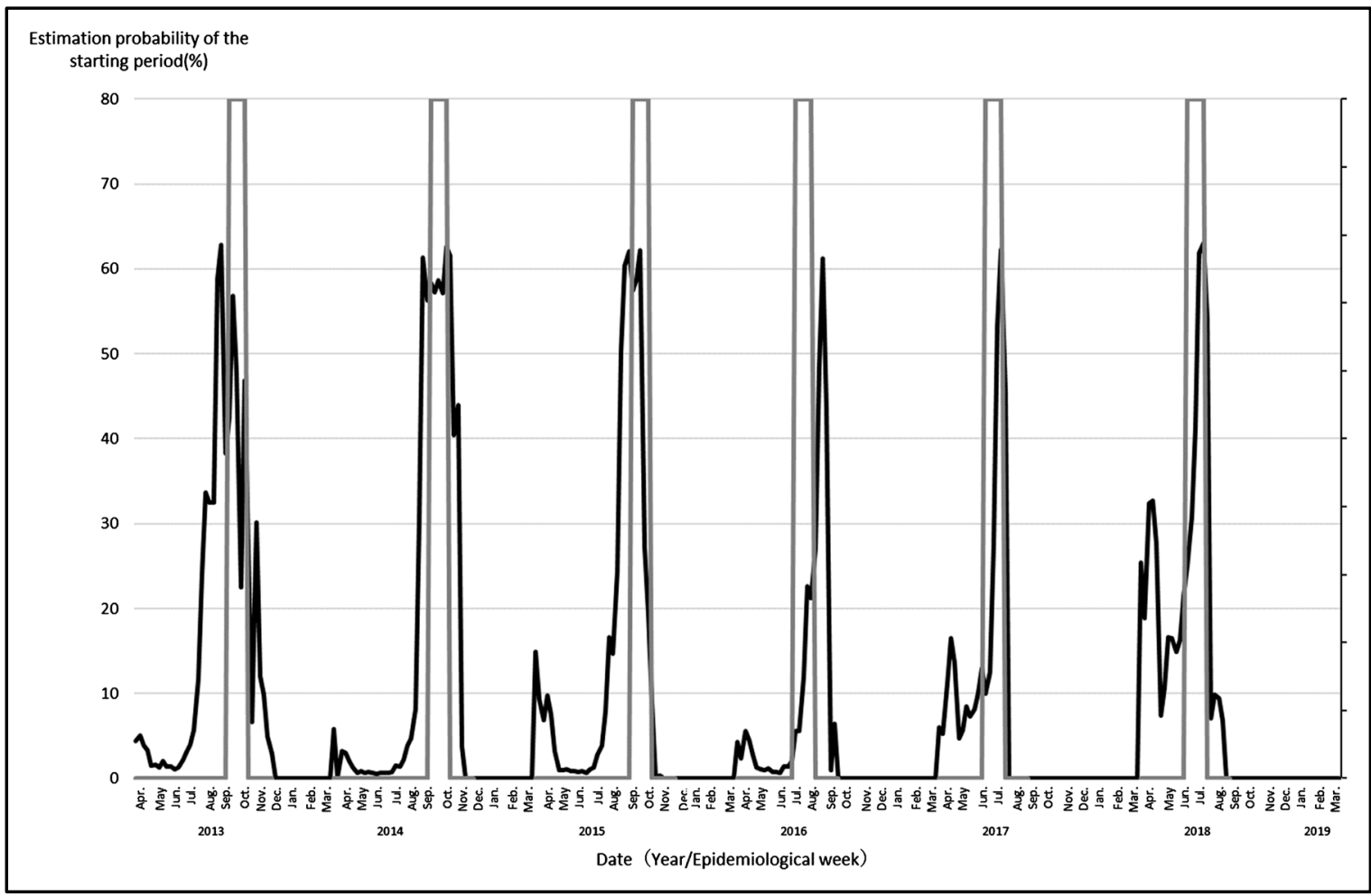

Note: The rectangular area indicate starting period in each season.

Figure 2. Estimated probability of the starting period in nationwide. 
Table 1. Results of the probability model for the starting period to start to take palivizumab in palivizumab nationwide and by prefecture by season.

\begin{tabular}{|c|c|c|c|c|c|c|c|}
\hline & Criterion & 2013 & 2014 & 2015 & 2016 & 2017 & 2018 \\
\hline Nationwide & 0.606132 & $\mathrm{~B}+$ & $\mathrm{B}+$ & $\mathrm{B}+$ & $\mathrm{A}$ & $\mathrm{H}-$ & $\mathrm{H}-$ \\
\hline Hokkaido & 0.365076 & B & A & $\mathrm{H}-$ & $\mathrm{B}+$ & H- & B \\
\hline Aomori & 0.4165 & B & B & B & B & $\mathrm{H}$ & $\mathrm{H}$ \\
\hline Iwate & 0.383186 & B & B & B & B & H- & A \\
\hline Miyagi & 0.399754 & B & $\mathrm{H}-$ & B & B & $\mathrm{H}$ & B \\
\hline Akita & 0.279561 & B & B & B & B & B & B \\
\hline Yamagata & 0.377601 & B & B & B & B & A & B \\
\hline Fukushima & 0.455593 & B & A & B & B & $\mathrm{B}+$ & B \\
\hline Ibaraki & 0.508223 & $\mathrm{H}$ & B & B & A & $\mathrm{H}-$ & A \\
\hline Tochigi & 0.469719 & B & B & B & B & $\mathrm{H}-$ & $\mathrm{H}$ \\
\hline Gunma & 0.433855 & B & B & B & B & B & B \\
\hline Saitama & 0.582684 & A & $\mathrm{B}+$ & $\mathrm{H}$ & A & A & $\mathrm{H}-$ \\
\hline Chiba & 0.539153 & $\mathrm{H}-$ & $\mathrm{B}+$ & $\mathrm{B}+$ & A & A & $\mathrm{H}-$ \\
\hline Tokyo & 0.58213 & $\mathrm{H}-$ & $\mathrm{B}+$ & A & A & $\mathrm{H}-$ & $\mathrm{H}-$ \\
\hline Kanagawa & 0.433825 & A & $\mathrm{B}+$ & $\mathrm{B}+$ & A & A & $\mathrm{H}-$ \\
\hline Niigata & 0.379723 & A & B & A & A & $\mathrm{H}-$ & B \\
\hline Toyama & 0.339192 & B & B & B & B & B & B \\
\hline Ishikawa & 0.395159 & B & B & B & B & B & B \\
\hline Fukui & 0.302659 & A & B & B & B & B & B \\
\hline Yamanashi & 0.493605 & B & B & B & B & B & B \\
\hline Nagano & 0.374563 & B & B & B & B & B & $\mathrm{H}-$ \\
\hline Gifu & 0.248725 & B & B & B & B & A & $\mathrm{H}$ \\
\hline Shizuoka & 0.566482 & $\mathrm{~B}+$ & $\mathrm{H}$ & B & $\mathrm{A}$ & A & A \\
\hline Aichi & 0.583744 & $\mathrm{H}$ & $\mathrm{B}+$ & $\mathrm{H}$ & A & A & $\mathrm{H}-$ \\
\hline Mie & 0.458855 & B & B & B & A & A & $\mathrm{H}$ \\
\hline Shiga & 0.45608 & B & B & B & B & B & B \\
\hline Kyoto & 0.35744 & B & B & B & B & A & $\mathrm{A}$ \\
\hline Osaka & 0.383502 & $\mathrm{H}$ & $\mathrm{H}-$ & $\mathrm{H}$ & A & A & $\mathrm{H}-$ \\
\hline Hyogo & 0.539787 & $\mathrm{H}-$ & $\mathrm{B}+$ & B & A & B & $\mathrm{H}-$ \\
\hline Nara & 0.415037 & B & B & B & B & B & B \\
\hline Wakayama & 0.231956 & B & B & B & B & B & B \\
\hline Tottori & 0.450884 & B & B & B & B & B & $\mathrm{B}$ \\
\hline Shimane & 0.285923 & $\mathrm{H}$ & B & B & B & B & A \\
\hline Okayama & 0.445476 & B & B & B & B & $\mathrm{H}$ & A \\
\hline Hiroshima & 0.268377 & A & B & $\mathrm{B}+$ & A & $\mathrm{H}$ & A \\
\hline Yamaguchi & 0.333154 & A & H- & B & A & $\mathrm{H}-$ & $\mathrm{B}+$ \\
\hline
\end{tabular}




\section{Continued}

\begin{tabular}{cccccccc}
\hline Tokushima & 0.474494 & B & B & B & B & H & H- \\
Kagawa & 0.476756 & B & B & B & B & B & B \\
Ehime & 0.352112 & H- & B & B & B & B & A \\
Kochi & 0.253556 & B & B & B & B & B & B \\
Fukuoka & 0.245601 & B & A & H- & B & B & B \\
Saga & 0.217094 & B & B & B & B & B & B \\
Nagasaki & 0.199576 & B & B & B & B & H & B \\
Kumamoto & 0.316938 & B & B & B & H & B & B \\
Oita & 0.293901 & A & B & B & B & B & H \\
Miyazaki & 0.290768 & B & B & B & B & B & B \\
Kagoshima & 0.381133 & H & B & B & B & B & B \\
Okinawa & 0.343533 & B+ & B+ & B+ & A & B & H \\
\hline
\end{tabular}

Note: " $\mathrm{H}$ " indicates that the probability model predicted starting period precisely, "B" and " $\mathrm{A}$ " indicates that the probability model predicted it before or after of starting period. "H-" indicates that the probability model predicted last two weeks in starting period. " $\mathrm{B}+$ " indicates that the probability model predicted during the two weeks before starting period. "Criterion" was defined the smallest probability among the highest probability in each season in each prefecture.

model predicted the starting period precisely. " $\mathrm{A}$ " and " $\mathrm{B}$ " indicate its prediction was before and after starting period, respectively. Moreover, when we consider the delay in NOSSID into consideration, even though the model predicted before the starting period, it predicated the starting date within two weeks before the starting period, we can start to take in the starting period. Therefore, though the model fails to predict the starting period precisely, patients can start to take within the starting period. We denote this result as " $\mathrm{B}+$." Conversely, even though the model predicted the starting period precisely, if it predicted the starting date in the last two weeks of the starting period, the patients cannot start to take within the starting period. We denote this result as "H-." Therefore, when we consider the delay in NOSSID into consideration, the patients can start to take in the case of the estimation results was " $\mathrm{B}+$ " or " $\mathrm{H}$ " but not " $\mathrm{H}-$."

In nationwide, criterion was estimated at about $60 \%$. The last two seasons, the model predicted precisely the starting period. However, the first three seasons, the initial date when the estimated probability was higher than criterion were before starting period. In 2016 season, the initial date when the estimated probability was higher than criterion was after starting period.

The criterions among prefectures varied from 0.1996 in Nagasaki to 0.5826 in Tokyo. In total, the model predicted precisely in 47 cases. Conversely, the initial date when the estimated probability was higher than criterion were before starting period in 195 cases. Moreover, in 46 cases, the initial date when the estimated probability was higher than criterion was after starting period.

The most precisely predicted season was 2018 and the model predicted precisely in 16 of 48 prefectures or nationwide. Conversely, the worst predicted season was 2016, the model predicted precisely only one of 48 prefectures or na- 
tionwide. There were many prefectures where the model failed to predict precisely in all six seasons.

Conversely, when we consider the delay in NOSSID into consideration, the patients in 10 prefectures can start to take in the starting period in 2014. The following year was 2013 and the next was 2015.

In total, the model predicted the starting period precisely in $16.6 \%$ of all year and prefectures pairs. When we consider the delay in NOSSID into consideration, the patients can start to take in $14.9 \%$ of all year and prefectures pairs.

\section{Discussion}

The criterion which is defined as the lowest probability among the highest probability in each season was the highest in nationwide and following in Tokyo. The lowest was in Nagasaki and it was lower than 0.2. The lower criterion itself means badly fitness of the model. Moreover, the proportion of the model predicted precisely was remained about $15 \%$. Unfortunately, the model failed to predict the starting period by prefecture in total.

The primarily reason might be that we applied the same formulation which is selected in nationwide to all prefecture, though using data and thus estimated coefficients were different for each prefecture. We might have to allow another specification in each prefecture. Moreover, though we defined the criterion as above, we have to define it more carefully with closing the situation in each prefecture. These examinations have to be our next challenge.

The secondly potential reason was shortcoming in NOSSID itself. Especially, it was weekly data, and thus it might not contain sufficient information to predict the starting period. In fact, some daily surveillance for RS virus infection such as ORCA surveillance [5] or (Nursery) School Absenteeism Surveillance System ((N)SASSy) [8]-[13] have sufficient power to predict the starting period using the similar model. If this reason was important, we have to give up developing the prediction model for the starting period in each prefecture. Because (N)SASSy or ORCA surveillance cannot provide sufficient information to predict in all prefectures, unfortunately.

\section{Conclusion}

We developed the prediction model for the starting period to take palivizumab. However, the results of the probability model were not good. Namely, the model predicted the starting period precisely only in $16.6 \%$. When we consider the delay in NOSSID into consideration, the patients can start to take it only in $14.9 \%$. Therefore, we have to develop more sophisticated model for prediction at prefecture level.

\section{Conflicts of Interest}

The authors declare no conflicts of interest regarding the publication of this paper. 


\section{References}

[1] Hall, C.B., Weinberg, G.A., Iwane, M.K., et al. (2009) The Burden of Respiratory Syncytial Virus Infection in Young Children. New England Journal of Medicine, 360, 588-598. https://doi.org/10.1056/NEJMoa0804877

[2] Lu, G., Gonzalez, R., Guo, L., et al. (2011) Large-Scale Seroprevalence Analysis of Human Metapneumovirus and Human Respiratory Syncytial Virus Infections in Beijing, China. Virology Journal, 8, 62. https://doi.org/10.1186/1743-422X-8-62

[3] Gerretsen, H.E. and Sande, C.J. (2017) Development of Respiratory Syncytial Virus (RSV) Vaccines for Infants. Journal of Infection, 74, S143-S146. https://doi.org/10.1016/S0163-4453(17)30205-0

[4] Kanou, K., Arima, Y., Kinoshita, H., et al. (2018) Respiratory Syncytial Virus Surveillance System in Japan: Assessment of Recent Trends, 2008-2015. Japanese Journal of Infectious Diseases, 71, 250-255. https://doi.org/10.7883/yoken.JJID.2017.261

[5] Ueno, T., Sugawara, T. and Ohkusa, Y. (2020) Proposal of the Probability Model for Starting Period to Initiate to Take Palivizumab Using ORCA Surveillance, Submitted.

[6] Ueno, T., Sugawara, T., Ohkusa, Y., et al. (2019) Retrospective Evaluation for ORCA Surveillance Comparison with Prescription Surveillance. Journal of Biosciences and Medicines, 7, 1-8. https://doi.org/10.4236/jbm.2019.712001

[7] Taniguchi, K., Hashimoto, S., Kawado, M., et al. (2007) Overview of Infectious Disease Surveillance System in Japan, 1999-2005. Journal of Epidemiology, 17, S3-S13. https://doi.org/10.2188/jea.17.S3

[8] Kurita, J., Nagasu, N., Nagata, N., et al. (2018) Outbreak of Human Metapneumovirus in Ibaraki, Japan and Its Descriptive Epidemiology. Health, 10, 749-757. https://doi.org/10.4236/health.2018.106057

[9] Kambe, C., Fujii, H., Niu, T., et al. (2018) Enhanced Surveillance for National (Handicapped) Sports Games in Wakayama, Japan 2015. Journal of Biosciences and Medicines, 6, 35-47. https://doi.org/10.4236/jbm.2018.67004

[10] Kurita, J., Sugawara, T., Matsumoto, K., et al. (2018) Association among (Nursery) School Absenteeism Surveillance System and Incidence of Infectious Diseases. School Health, 14, 21-27.

[11] Kurita, J., Nagasu, N., Nagata, N., et al. (2018) Descriptive Epidemiology for $M y$ coplasma pneumoniae Infection Using (Nursery) School Absenteeism Surveillance System and Proposal for Countermeasures. Journal of Biosciences and Medicines, 6 , 33-42. https://doi.org/10.4236/jbm.2018.610005

[12] Sugishita, Y., Sugawara, T. and Ohkusa, Y. (2019) Association of Influenza Outbreak in Each Nursery School and Community in a Ward in Tokyo, Japan. Journal of Infection and Chemotherapy, 25, 695-701. https://doi.org/10.1016/j.jiac.2019.03.010

[13] Tanabe, Y., Kurita, J., Nagasu, N., et al. (2019) Infection Control in Nursery Schools and Schools Using a School Absenteeism Surveillance System. The Tohoku Journal of Experimental Medicine, 247, 173-178. https://doi.org/10.1620/tjem.247.173 